\title{
MODULAR GROUP ALGEBRAS OF $N$-GROUPS
}

\author{
WILLIAM ULLERY
}

(Communicated by Bhama Srinivasan)

\begin{abstract}
For a prime $p$, let $F$ be the field with $p$ elements and let $G$ be a reduced $p$-primary abelian $N$-group. If $F G \cong F H$ for some group $H$, then it is shown that $G \cong H$.
\end{abstract}

Let $F$ be a field of characteristic $p>0$ and let $G$ be an (infinite) abelian $p$-group. The question of whether an $F$-algebra isomorphism $F G \cong F H$ for some group $H$ implies $G \cong H$ has been considered by Berman and Mollov [1], and by May [6, $\mathbf{7}, \mathbf{8}]$. It is shown in $[\mathbf{1}, \mathbf{6}]$, and by Dubois and Sehgal $[\mathbf{3}]$ that $F G \cong F H$ implies that the Ulm-Kaplansky invariants of $G$ and $H$ must be equal. Thus, the case when $G$ is countable can be settled with no major difficulties. In [1], the case when $G$ is a direct sum of cyclic groups is answered affirmatively. More generally, an affirmative answer was obtained if $G$ is a direct sum of countable groups (d.s.c.) in [7]. Recently, these results were generalized in [8] to totally projective groups (of arbitrary length); i.e., $F G \cong F H$ and $G$ totally projective implies $G \cong H$. In this paper, we generalize the result in [7] for d.s.c.'s in another direction. Here we will consider the case when $G$ is an $N$-group, a certain distinguished isotype subgroup of a d.s.c. $N$-groups were first introduced in Hill [5]. For the sake of the uninitiated reader, we include the definition below.

1. Preliminaries. Before stating our result in detail, we introduce some notation. First, all groups considered will be abelian and written multiplicatively. The letters $G, H, A$, and $B$ will always denote $p$-primary abelian groups. For abelian group terminology not defined herein, the reader is referred to Fuchs [4].

For an ordinal $\sigma$, we write $G^{p^{\sigma}}$ for the elements of $G$ with $p$-height $\geq \sigma$. If $g \in G^{p^{\sigma}} \backslash G^{p^{\sigma+1}}$ we write $h_{G}(g)=\sigma$. If no such $\sigma$ exists, we set $h_{G}(g)=\infty$ with the convention that $\infty>\sigma$ for all ordinals $\sigma$. Sometimes we may write $h(g)$ for $h_{G}(g)$ if the group $G$ is clear from the context. Suppose $G$ is a subgroup of the $p$-group $A$. Recall that $G$ is an isotype subgroup of $A$ if and only if $h_{G}(g)=h_{A}(g)$ for all $g \in G$.

A subgroup $G$ of a d.s.c. $p$-group $A$ is called an $N$-group (with containing d.s.c. $A$ ) if it satisfies the following three conditions:

(i) $G$ is isotype in $A$.

(ii) $(A / G)^{p^{\sigma}}=A^{p^{\sigma}} G / G$ if $\sigma<\Omega$, the first uncountable ordinal, and $(A / G)^{p^{\Omega+1}}$ $=1$.

(iii) $A / G$ is totally projective.

In Hill's paper [5], it was shown that two $N$-groups $G$ and $H$ are isomorphic if the

Received by the editors April 7, 1987.

1980 Mathematics Subject Classification (1985 Revision). Primary 20K10; Secondary 16A27.

Key words and phrases. $N$-groups, modular group algebras. 
socles $G[p]$ and $H[p]$ are isomorphic as valuated vector spaces (where the valuations are the height functions in $G$ and $H$ restricted to the socles). Note that if condition (ii) were replaced by the condition that $G$ is nice in $A$, then $G$ would be a direct summand of $A$, and hence itself a d.s.c. It is in this sense that one may consider $N$-groups to be "almost d.s.c.'s".

We now come to the statement of our main result.

THEOREM. For a prime $p$, suppose $F$ is the field with $p$ elements and $G$ is a p-group. If $G$ is a reduced $N$-group and $F G \cong F H$ for some group $H$, then $G \cong H$.

The proof of the theorem, which is given in $\S 3$, consists of two parts: First, we show that $H$ must be an $N$-group using a method developed in $\S 2$. Next, we use a result from Beers, Richman, and Walker [2] to show that the respective valuated socles are isomorphic.

2. Certain subgroups of units. If $F$ is a field of characteristic $p \neq 0$ and $A$ is a $p$-group, let $U(F A)$ be the set of elements with augmentation 1 (i.e. with coefficients summing to 1$)$. Note that $U(F A)$ is a p-group containing $A$ as a subgroup and is itself a subgroup of the group of units of $F A$. Note that if $F$ is assumed perfect, $U(F A)^{p^{\sigma}}=U\left(F A^{p^{\sigma}}\right)$ for all ordinals $\sigma$. Thus, for $\alpha \in U(F A), h_{U(F A)}(\alpha)=$ $\min \left\{h_{A}(a) \mid a \in \operatorname{supp}(\alpha)\right\}$ where $\operatorname{supp}(\alpha)$ is the support of $\alpha$; i.e., those $a \in A$ appearing nontrivially in $\alpha$.

Let $I(A)=\{\alpha \in F A \mid \operatorname{aug}(\alpha)=0\}$; i.e., $I(A)$ is the ideal of $F A$ consisting of all elements of augmentation 0 . Note that if $G \leq A$, then $1+F A \cdot I(G)$ is a subgroup of $U(F A)$ containing $G$ with $A \cap(1+F A \cdot I(G))=G$. This section is devoted to a study of $1+F A \cdot I(G)$. In particular, we shall see that some properties of the containment $G \leq A$ are shared by $1+F A \cdot I(G) \leq U(F A)$. Even though we shall continue to work with fields, we note that all results in this section remain true for commutative rings of characteristic $p$ which are perfect (i.e., every element is a $p$ th power). The proofs are identical to the ones given.

LEMMA 1. Suppose $F$ is a perfect field of characteristic $p \neq 0$ and $G$ is a subgroup of the p-group $A$. Then $G$ is isotype in $A$ if and only if $1+F A \cdot I(G)$ is isotype in $U(F A)$.

Proof. First suppose $G$ is isotype in $A$, and for some ordinal $\sigma, 1 \neq \alpha \in$ $U(F A)^{p^{\sigma}} \cap(1+F A \cdot I(G))$. Since $\alpha \in 1+F A \cdot I(G)$, there exist nonzero $r_{1}, \ldots, r_{n} \in$ $F$; nonidentity elements $g_{1}, \ldots, g_{n} \in G$; and $a_{1}, \ldots, a_{n} \in A$ with $\alpha=1+$ $\sum_{i=1}^{n} r_{i} a_{i}\left(g_{i}-1\right)$. We need to show that $\alpha \in 1+F A^{p^{\sigma}} \cdot I\left(G^{p^{\sigma}}\right)$. We do this by induction on $n$.

First, if $n=1$ or if $a_{1} g_{1}, a_{1}, a_{2} g_{2}, a_{2}, \ldots, a_{n} g_{n}, a_{n}$ are all in supp $\alpha$, then $a_{i} g_{i} \in$ $A^{p^{\sigma}}$ and $a_{i} \in A^{p^{\sigma}}$ for all $i, 1 \leq i \leq n$. Thus, $g_{i} \in A^{p^{\sigma}} \cap G=G^{p^{\sigma}}$ since $G$ is isotype in $A$. Thus $\alpha \in 1+F A^{p^{\sigma}} \cdot I\left(G^{p^{\sigma}}\right)$ and we may assume that $n>1$ and consider only two cases:

Case 1. Some $a_{i} g_{i}$ is not in $\operatorname{supp}(\alpha)$. In this case, there exist distinct subscripts $j_{1}, \ldots, j_{m}, j_{m+1}, \ldots, j_{m+s}$ not equal to $i$ satisfying the following two conditions:

(1) $a_{j_{k}} g_{j_{k}}=a_{j_{m+t}}=a_{i} g_{i}$ for all $k$ and $t(1 \leq k \leq m, 1 \leq t \leq s)$.

(2) $r_{i} a_{i} g_{i}+\sum_{k=1}^{m} r_{j_{k}} a_{j_{k}} g_{j_{k}}-\sum_{t=1}^{s} r_{j_{m+t}} a_{j_{m+t}}=0$.

Set $\beta=r_{i} a_{i}\left(g_{i}-1\right)+r_{j_{1}} a_{j_{1}}\left(g_{j_{1}}-1\right)+\cdots+r_{j_{m+s}} a_{j_{m+s}}\left(g_{j_{m+s}}-1\right)$. Note that $\beta$ is a sum of $m+s+1$ terms of the form $r a(g-1), r \in F, a \in A, g \in G$. Thus, to 
complete the induction in this case, it suffices to show that $\beta$ can be written as a sum of $m+s$ terms of this form.

Now, using conditions (1) and (2) we have

$$
\begin{aligned}
\beta= & -r_{i} a_{i}-r_{j_{1}} a_{j_{1}}-\cdots-r_{j_{m}} a_{j_{m}}+r_{j_{m+1}} a_{j_{m+1}} g_{j_{m+1}}+\cdots+r_{j_{m+s}} a_{j_{m+s}} g_{j_{m+s}} \\
= & -r_{i} a_{i}-r_{j_{1}} a_{i} g_{i} g_{j_{1}}^{-1}-\cdots-r_{j_{m}} a_{i} g_{i} g_{j_{m}}^{-1} \\
& +r_{j_{m+1}} a_{i} g_{i} g_{j_{m+1}}+\cdots+r_{j_{m+s}} a_{i} g_{i} g_{j_{m+s}} .
\end{aligned}
$$

Since the left-hand side of condition (2) has augmentation $0, r_{i}=-r_{j_{1}}-\cdots-r_{j_{m}}+$ $r_{j_{m+1}}+\cdots+r_{j_{m+s}}$. Thus, $-r_{i} a_{i}=r_{j_{1}} a_{i}+\cdots+r_{j_{m}} a_{i}-r_{j_{m+1}} a_{i}-\cdots-r_{j_{m+s}} a_{i}$. Consequently,

$$
\begin{aligned}
\beta= & -r_{j_{1}} a_{i}\left(g_{i} g_{j_{1}}^{-1}-1\right)-\cdots-r_{j_{m}} a_{i}\left(g_{i} g_{j_{m}}^{-1}-1\right) \\
& +r_{j_{m+1}} a_{i}\left(g_{i} g_{j_{m+1}}-1\right)+\cdots+r_{j_{m+s}} a_{i}\left(g_{i} g_{j_{m+s}}-1\right),
\end{aligned}
$$

a sum of $m+s$ terms of the desired form. As observed earlier, this completes Case 1.

Case 2. Some $a_{i}$ is not in $\operatorname{supp}(\alpha)$. In this case, we get obvious analogs for the conditions (1) and (2) in Case 1, and the argument follows along similar lines. We omit the details.

Conversely, suppose $K=1+F A \cdot I(G)$ is an isotype subgroup of $U=U(F A)$. Then, for all ordinals $\sigma, G \cap A^{p^{\sigma}} \leq K \cap U^{p^{\sigma}}=K^{p^{\sigma}}=1+F A^{p^{\sigma}} \cdot I\left(G^{p^{\sigma}}\right)$. Therefore, $G \cap A^{p^{\sigma}}=G \cap\left(A^{p^{\sigma}} \cap K^{p^{\sigma}}\right)=G \cap G^{p^{\sigma}}=G^{p^{\sigma}}$, showing $G$ is isotype in $A$.

In the proof of our next result, it will be convenient to have a definition. Suppose $\phi: A \rightarrow B$ is an epimorphism of $p$-groups. For an ordinal $\sigma$, call $\phi \sigma$-nice if for every $a \in A$ with $h_{B}(\phi(a)) \geq \sigma$, there exists $a_{0} \in A$ such that $\phi\left(a_{0}\right)=\phi(a)$ and $h_{A}\left(a_{0}\right) \geq \sigma$. If $K=\operatorname{Ker} \phi$, note that $\phi$ is $\sigma$-nice if and only if $(A / K)^{p^{\sigma}}=A^{p^{\sigma}} K / K$.

LEMMA 2. Suppose $F$ is a perfect field of characteristic $p \neq 0$ and suppose $\sigma$ is an ordinal. An epimorphism $\phi: A \rightarrow B$ of p-groups is $\sigma$-nice if and only if the induced map $\phi^{*}: U(F A) \rightarrow U(F B)$ is $\sigma$-nice.

PROOF. First suppose $\phi$ is $\sigma$-nice and $\alpha \in U(F G)$ is said that $h\left(\phi^{*}(\alpha)\right) \geq \sigma$. There exist $\alpha_{1}, \ldots, \alpha_{n}, \alpha_{n+1}, \ldots, \alpha_{r} \in F A$ satisfying the following three properties:

(I) $\alpha=\sum_{i=1}^{r} \alpha_{i}$.

(II) If $a \in \operatorname{supp}\left(\alpha_{i}\right), a^{\prime} \in \operatorname{supp}\left(\alpha_{j}\right)(1 \leq i \leq j \leq r), \phi(a)=\phi\left(a^{\prime}\right)$ if and only if $i=j$.

(III) $\operatorname{aug}\left(\alpha_{i}\right) \neq 0$ for $1 \leq i \leq n$, and $\operatorname{aug}\left(\alpha_{i}\right)=0$ for $n+1 \leq i \leq r$.

For each $i, 1 \leq i \leq n$, select $a_{i} \in \operatorname{supp}\left(\alpha_{i}\right)$. Then, $\phi^{*}(\alpha)=\sum_{i=1}^{n}\left(\operatorname{aug}\left(\alpha_{i}\right)\right) \phi\left(a_{i}\right)$. Since $h\left(\phi^{*}(\alpha)\right) \geq \sigma, h\left(\phi\left(a_{i}\right)\right) \geq \sigma$ for all $i, 1 \leq i \leq n$. Thus, since $\phi$ is $\sigma$ nice, there exist $a_{10}, \ldots, a_{n 0} \in A^{p^{\sigma}}$ with $\phi\left(a_{i 0}\right)=\phi\left(a_{i}\right), 1 \leq i \leq n$. Set $\sigma_{0}=$ $\sum_{i=1}^{n}\left(\operatorname{aug}\left(\alpha_{i}\right)\right) a_{i 0}$. Then, $\phi^{*}\left(\alpha_{0}\right)=\phi^{*}(\alpha)$ and $h\left(\alpha_{0}\right) \geq \sigma$, showing $\phi^{*}$ is $\sigma$-nice.

Conversely, suppose $\phi^{*}$ is $\sigma$-nice and $a \in A$ is such that $h(\phi(a)) \geq \sigma$. Since $\phi=\phi^{*} \mid A$ and $A$ is isotype in $U(F A)$, there exists $\alpha \in U(F A)$ such that $\phi^{*}(\alpha)=$ $\phi(a)$ and $h(\alpha) \geq \sigma$. Clearly there must be some $a_{0} \in \operatorname{supp}(\alpha)$ with $\phi\left(a_{0}\right)=\phi(a)$. Moreover, $a_{0} \in \operatorname{supp}(\alpha)$ implies $h\left(a_{0}\right) \geq h(\alpha) \geq \sigma$. Therefore, $\phi$ is $\sigma$-nice.

We now give several corollaries. In all of these, $F$ will be a perfect field of characteristic $p \neq 0$, and $G$ will be a subgroup of the $p$-group $A$. Also, set $K=$ $1+F A \cdot I(G)$ and $U=U(F A)$. 
COROLlaRY 1. For an ordinal $\sigma,(A / G)^{p^{\sigma}}=A^{p^{\sigma}} G / G$ if and only if $(U / K)^{p^{\sigma}}=$ $U^{p^{\sigma}} K / K$.

ProOF. The quotient map $A \rightarrow A / G$ induces $U(F A) \rightarrow U(F(A / G))$ with kernel $K=1+F A \cdot I(G)$. Thus the result follows from Lemma 2 and the remark preceding it.

The next result was proven independently in [8]. It follows directly from our Corollary 1.

\section{COROllary 2. $G$ is nice in $A$ if and only if $K$ is nice in $U$.}

Recall that a subgroup is called balanced if it is both nice and isotype. From Lemma 1 and Corollary 2, we get

COROLlaRY 3. $G$ is a balanced subgroup of $A$ if and only if $K$ is a balanced subgroup of $U$.

Finally, we arrive at the main result of this section. This is the key result needed in the proof of our Theorem.

PROPOSITION. Suppose $F$ is a perfect field of characteristic $p \neq 0$, and suppose $G$ is a subgroup of the d.s.c. p-group $A$. Then, $G$ is an $N$-group with containing d.s.c. $A$ if and only if $1+F A \cdot I(G)$ is an $N$-group with containing d.s.c. $U(F A)$.

ProOF. First observe that $U(F A)$ is a d.s.c. by [7, Theorem 2]. Set $U=U(F A)$ and $K=1+F A \cdot I(G)$. Now, by Lemma $1, G$ is isotype in $A$ if and only if $K$ is isotype in $U$. By Corollary $1,(A / G)^{p^{\sigma}}=A^{p^{\sigma}} G / G$ for all $\sigma<\Omega$ if and only if $(U / K)^{p^{\sigma}}=U^{p^{\sigma}} K / K$ for all $\sigma<\Omega$. Moreover, since $U / K \cong U(F(A / G))$ and $U(F(A / G))^{p^{\Omega+1}}=U\left(F(A / G)^{p^{\Omega+1}}\right)$, it is clear that $(A / G)^{p^{\Omega+1}}=1$ if and only if $(U / K)^{p^{\Omega+1}}=1$. Finally, $A / G$ is totally projective if and only if $U(F(A / G)) \cong U / K$ is totally projective by $[8$, Theorem 1$]$. Thus $G \leq A$ satisfies conditions (i), (ii), and (iii) in the definition of an $N$-group if and only if $K \leq U$ does.

3. Proof of the Theorem. Since $F G \cong F H$, there is an augmentation preserving isomorphism $F G \rightarrow F H$. As a consequence, $U(F G)$ contains an $F$-basis for $F G$ isomorphic to $H$. Thus, we may assume $F G=F H, U(F G)=U(F H)$, and $I(G)=I(H)$.

Suppose $B$ is a containing d.s.c. for the $N$-group $G$. Because $G$ is reduced and $(B / G)^{p^{\Omega+1}}=1, B$ is also reduced. By [7, Theorem 2], we conclude that $U(F B)=$ $B \times L$ where $L$ is a reduced d.s.c. Since $L^{p^{\Omega+1}}=1$, it follows that $A=U(F B)$ is also a containing d.s.c. for $G$. Moreover, $A$ also contains $H$. Therefore, by the Proposition, $1+F A \cdot I(G)=1+F A \cdot I(H)$ is an $N$-group with containing d.s.c. $U(F A)$. A further application of the Proposition shows that $H$ is also an $N$-group with containing d.s.c. $A$.

Let $\lambda: F G \rightarrow F G$ (respectively, $\lambda^{\prime}: F H \rightarrow F H$ ) be the Frobenius endomorphism of $F G$ (respectively, $F H$ ); i.e., $\lambda(\alpha)=\alpha^{p}$ for all $\alpha \in F G$. By [2, Theorem 3.1], there is an isomorphism $F \otimes G[p] \rightarrow \operatorname{Ker} \lambda /(I(G) \cdot \operatorname{Ker} \lambda)$ which restricts to an isomorphism

$$
F \otimes G^{p^{\sigma}}[p] \rightarrow\left(\left((\operatorname{Ker} \lambda) \cap F G^{p^{\sigma}}\right)+I(G) \cdot \operatorname{Ker} \lambda\right) /(I(G) \cdot \operatorname{Ker} \lambda)
$$

for every ordinal $\sigma$. Since $\operatorname{Ker} \lambda=\operatorname{Ker} \lambda^{\prime}, F G^{p^{\sigma}}=F H^{p^{\sigma}}$, and $I(G)=I(H)$, we see that there is an isomorphism $F \otimes G[p] \rightarrow F \otimes H[p]$ that restricts to an isomorphism 
$F \otimes G^{p^{\sigma}}[p] \rightarrow F \otimes H^{p^{\sigma}}[p]$ for every ordinal $\sigma$. Finally, since $|F|=p$, there are isomorphisms $G[p] \rightarrow F \otimes G[p]$ and $H[p] \rightarrow F \otimes H[p]$ which restrict to isomorphisms $G^{p^{\sigma}}[p] \rightarrow F \otimes G^{p^{\sigma}}[p]$ and $H^{p^{\sigma}}[p] \rightarrow F \otimes H^{p^{\sigma}}[p]$, respectively. Consequently, there exists a height-preserving isomorphism of $G[p]$ onto $H[p]$. Therefore, $H \cong G$ by [5, Corollary 1]. This completes the proof of the Theorem.

\section{REFERENCES}

1. S. D. Berman and T. Zh. Mollov, The group rings of abelian p-groups of any cardinality, Mat. Zametki 6 (1969), 381-392; Math. Notes 6 (1969), 686-692. (Russian)

2. D. Beers, F. Richman, and E. A. Walker, Group algebgras of abelian groups, Rend. Sem. Mat. Univ. Padova 69 (1983), 41-50.

3. P. F. Dubois and S. K. Sehgal, Another proof of the invariance of Ulm's functions in commutative modular group rings, Math. J. Okayama Univ. 15 (1972), 137-139.

4. L. Fuchs, Infinite abelian groups, vols. I and II, Academic Press, New York, 1970 and 1973.

5. P. Hill, The classification of $N$-groups, Houston J. Math. 10 (1984), 43-55.

6. W. May, Commutative group algebras, Trans. Amer. Math. Soc. 136 (1969), 139-149.

7. __ Modular group algebras of totally projective p-primary groups, Proc. Amer. Math. Soc. 76 (1979), 31-34.

8. _ـ Modular group algebras of simply presented abelian groups, preprint.

Department of MATHEMATICS, UNIVERsity OF KANSAS, LAWRENCE, Kansas 66045 36849

Department of Mathematics aCA, Auburn University, Auburn, Alabama 